\title{
Enterococcus faecalis strains show culture heterogeneity in cell surface charge
}

\author{
Correspondence \\ Bastiaan P. Krom \\ b.p.krom@med.umcg.nl
}

Received 23 August 2005

Revised 16 November 2005

Accepted 16 November 2005

\author{
Annet E. J. van Merode, ${ }^{1}$ Henny C. van der Mei, ${ }^{1}$ Henk J. Busscher, ${ }^{1}$ \\ Karola Waar ${ }^{2}$ and Bastiaan P. Krom ${ }^{1}$
}

Departments of Biomedical Engineering ${ }^{1}$ and Medical Microbiology ${ }^{2}$, University Medical Center Groningen, and University of Groningen, Antonius Deusinglaan 1, 9713 AV Groningen, The Netherlands

\begin{abstract}
Adhesion of micro-organisms to biotic and abiotic surfaces is an important virulence factor and involves different types of interactions. Enterococcus faecalis, a human commensal and an important opportunistic pathogen, has the ability to adhere to surfaces. Biliary stents frequently become clogged with bacterial biofilms, with $E$. faecalis as one of the predominant species. Six E. faecalis strains isolated from clogged biliary stents were investigated for the presence of specific biochemical factors involved in their adhesion: aggregation substances (Aggs) and the enterococcal surface protein (encoded by the esp gene). In addition, physico-chemical factors involved in adhesion (zeta potential and cell surface hydrophobicity) were determined, as well as the influence of ox bile on these properties. Two-thirds of the biliary stent isolates displayed culture heterogeneity in the $\mathrm{pH}$ dependence of their zeta potentials. Moreover, 24 out of 46 clinical isolates of $E$. faecalis, including 11 laboratory strains, also displayed such heterogeneity. The culture heterogeneity was demonstrated to be a stable trait, not caused by quorum sensing, not plasmid mediated, and independent of the presence of esp and Agg. Data presented show that culture heterogeneity in zeta potential enhances adhesion to an abiotic surface. A higher prevalence of culture heterogeneity in zeta potential in pathogenic as compared to non-pathogenic isolates could indicate that this phenomenon might play a role in virulence and putatively in pathogenesis.
\end{abstract}

\section{INTRODUCTION}

The opportunistic pathogen Enterococcus faecalis, a member of the commensal human flora, is among the leading causes of nosocomial infection (Low et al., 2001). Several traits of E. faecalis are described as virulence factors (reviewed by Kayaoglu \& Østavik, 2004), and adhesion to biotic and abiotic surfaces is one of them. E. faecalis is one of the predominant organisms found in biofilms in clogged biliary stents, used to palliate obstructions of the biliary and pancreatic ducts (Dowidar et al., 1991). E. faecalis can translocate from the duodenum to colonize the stent (Di Rosa et al., 1999), where it encounters a high concentration of bile.

Factors specific for E. faecalis involved in adhesion include adhesins (e.g. the enterococcal surface protein, Esp) and aggregation substances (Aggs) (reviewed by Kayaoglu \& Østavik, 2004). Esp was found to be enriched in infectionderived enterococcal strains (Shankar et al., 1999) and it is associated with enhanced adhesion to abiotic surfaces and

Abbreviations: Agg, aggregation substance; Esp, enterococcal surface protein; RAPD, random amplified polymorphic DNA.

Additional tables of zeta potential distributions, and a figure showing that sonication had no effect on zeta potential distributions, are provided as supplementary data with the online version of this paper. biofilm formation by E. faecalis (Toledo-Arana et al., 2001; Tendolkar et al., 2004). Aggregation substances (Aggs) are plasmid-encoded surface proteins involved in conjugation, although a gene encoding an Agg was identified on a pathogenicity island of E. faecalis (Shankar et al., 2001a). Several different Aggs have been identified, and all are expressed in response to pheromones, resulting in formation of aggregates of donor and recipient cells and transfer of plasmids (Clewell, 1993). Waar et al. (2002c) found that E. faecalis strains expressing Esp and Agg adhered better to hydrophobic bile drain materials.

The favourable interactions between specific adhesion sites are confined to localized sites on the cell surface. The interactions originating from the entire cell body are usually referred to as non-specific interactions, although both specific and non-specific interactions are mediated by the same fundamental physico-chemical forces (Van Oss, 2003), including Lifshitz-Van der Waals, electrostatic and acidbase interactions (Van Loosdrecht et al., 1987). In general, investigation of bacterial cell surface properties determines average properties of an entire culture rather than distinguishing between individual cells. Specific interactions are short-ranged, but strong as compared with the non-specific interactions (Van Oss, 2003). Hence the latter 
are responsible for initial, reversible adhesion. Since all surfaces occurring in nature carry a net negative charge, electrostatic interactions in bacterial adhesion are nearly always repulsive and have to be overcome by attractive Lifshitz-Van der Waals, hydrophobic and specific interaction forces. E. faecalis strains grown in the presence of ox bile, for instance, were generally more hydrophobic and more negatively charged than after growth in the absence of bile, and accordingly adhered better to abiotic surfaces (Waar et al., 2002b). In the measurement of bacterial cell surface charge or hydrophobicity, or any other property, bacterial cultures are usually considered as populations of identical organisms, although it is known that several strains display distinct subpopulations even in pure cultures. In these cultures, the distribution of properties of the cells is more heterogeneous than normally assumed, which goes beyond differences in, for example, cell size. Different subpopulations within one culture can differ in flagellation (Streger et al., 2002), natural competence (Dubnau, 1991), autofluorescence (Kell et al., 1991), or cell surface charge (Cowan et al., 1992).

With microelectrophoresis it is possible to study individual cells with respect to their surface charge or zeta potential (Glynn et al., 1998; Van der Mei \& Busscher, 2001; Wilson et al., 2001). The zeta potential is correlated with the charge on the bacterial cell surface by the nature and number of ionizable groups exposed on the surface and depends amongst other factors on $\mathrm{pH}$ and ionic strength. In the case of a heterogeneous population, the zeta potential distribution measured may either be an extremely wide, usually Gaussian distribution or display separate Gaussian distributions (Geertsema-Doornbusch et al., 1994; Noordmans et al., 1993; Streger et al., 2002; Glynn et al., 1998). Often fresh clinical isolates display greater culture heterogeneity than laboratory strains kept in stock for many years.

In the current study, E. faecalis strains isolated from clogged biliary stents were investigated for the presence of factors involved in adhesion to abiotic surfaces. It was found that four of six isolates were heterogeneous with respect to zeta potential and that this heterogeneity was not related to the presence of esp or Agg, to quorum sensing, or to growth medium and growth phase. Furthermore, the data suggested that culture heterogeneity in zeta potential enhances adhesion to an abiotic surface. Analysis of the zeta potential distribution of 46 clinical isolates indicated that culture heterogeneity in zeta potential is a common phenomenon and might serve an important role during virulence and pathogenesis.

\section{METHODS}

Strains and growth conditions. Six E. faecalis strains (BS385, BS4126, BS937, BS1037, BS11297 and BS12297) were isolated from clogged polyethylene biliary stents, explanted from separate patients. The bacteria were identified using Biolog GP plates and stored in $7 \%$ dimethyl sulfoxide at $-80^{\circ} \mathrm{C}$. E. faecalis strains isolated from the faeces of healthy volunteers, liver transplant patients and blood cultures have been described and characterized for the presence of Esp and Agg elsewhere (Waar et al., 2002a). The enterococcal laboratory strains used in this study were: E. faecalis JH2-2 (Jacob \& Hobbs, 1974), OG1X (Ike et al., 1983), OG1X(pAD1) (Clewell et al., 1982), OG1XE(pAD1) (Muscholl et al., 1993), OG1X(pAM373) (Clewell et al., 1985), FA2-2 (Clewell et al., 1982), FA2-2(pAD1) (Chow et al., 1993), MMH594 (Shankar et al., 1999), MMH594b (Shankar et al., 2001b), OG1RF (Dunny et al., 1978) and OG1RF(pCF10) (Dunny et al., 1981). All isolates were cultured from frozen stock on blood agar plates. Precultures were grown in $3 \mathrm{ml}$ brain heart infusion (BHI, Oxoid) overnight aerobically at $37^{\circ} \mathrm{C}$. Cultures were grown from precultures in $200 \mathrm{ml} \mathrm{BHI}$ with or without $50 \mathrm{mg} \mathrm{ml}^{-1}$ ox bile (Merck) overnight at $37^{\circ} \mathrm{C}$.

Genetic analysis. E. faecalis strains isolated from clogged biliary stents were evaluated for genetic relatedness using PFGE and random amplified polymorphic DNA (RAPD). PFGE was performed as described by Murray et al. (1990). Chromosomal DNA was digested with SmaI. RAPD was performed as described by Barbier et al. (1996) using the following primers: ERIC-1, 5'-ATGTAAGCTCCTGGGGATTCAC-3'; ERIC-2, 5' -AAGTAAGTGACTGGGGTGAGCG-3'; 23SR, 5'-GGTACCTTAGATGTTTCAGTTC-3'; and 16SR, 5' -TTGTACACACCGCCCGTCA-3'.

Detection of esp and Agg. The presence of the esp gene in the E. faecalis strains isolated from clogged biliary stents was determined by PCR amplification using the primers esp11 and esp12 as described by Shankar et al. (1999).

The presence of Aggs was detected in a clumping assay as described elsewhere (Dunny et al., 1978) with some modifications. The supernatant of an overnight culture of the pheromone-producing strain E. faecalis JH2-2 (Jacob \& Hobbs, 1974) grown in BHI was autoclaved and used as a source of pheromones. A $2 \mathrm{ml}$ volume of fresh BHI was mixed with $0.5 \mathrm{ml}$ of the supernatant, and $25 \mu$ of overnight cultured enterococci was added. The mixture was grown for $4 \mathrm{~h}$ at $37^{\circ} \mathrm{C}$ in a rotating device at 65 r.p.m. and examined for the formation of aggregates.

Physico-chemical cell surface properties. The hydrophobicity of the E. faecalis strains isolated from clogged biliary stents were assessed by water contact angle measurements by employing the sessile drop technique on bacteria deposited on membrane filters. Briefly, the bacteria from a $200 \mathrm{ml}$ overnight culture were harvested by centrifugation $\left(6500 \mathrm{~g}, 5 \mathrm{~min}, 10^{\circ} \mathrm{C}\right)$, washed twice and resuspended in demineralized water, and deposited on a $0.45 \mu \mathrm{m}$ pore-size filter (Millipore) using negative pressure. A lawn of approximately 50 stacked layers of bacteria was produced on the filter. The filters were dried for $30 \mathrm{~min}$ in order to measure plateau water contact angles. Measurements were performed in triplicate with separately cultured bacteria, with six water droplets measured on each filter.

Zeta potential distributions of all strains were determined by microelectrophoresis. The strains were grown overnight, harvested by centrifugation $\left(6500 \mathrm{~g}, 5 \mathrm{~min}, 10^{\circ} \mathrm{C}\right)$ and washed twice with $10 \mathrm{mM}$ potassium phosphate $\mathrm{pH} 7 \cdot 0$. Zeta potential distributions were measured in $10 \mathrm{mM}$ potassium phosphate $\mathrm{pH} \mathrm{2,} \mathrm{3,} \mathrm{4,} \mathrm{5,} 7$ and 9 with a Lazer Zee Meter 501 (PenKem), equipped with image analysis options for zeta sizing. Briefly, the microelectrophoresis chamber was filled with a bacterial suspension with a density of $10^{7}-$ $10^{8}$ cells $\mathrm{ml}^{-1}$ and a voltage difference of $150 \mathrm{~V}$ was applied over the chamber. The velocity of each individual bacterium was determined by image sequence analysis and from this its zeta potential was calculated, assuming that the Helmholtz-Smoluchowski equation held. Zeta potential distributions were measured in triplicate with separately cultured bacteria. 


\section{Stability of the subpopulations}

Serial passaging. Four biliary stent isolates were serially passaged up to 50 times in liquid medium to test the stability of the subpopulations. Passaging of the strains consisted of a daily repetitive inoculation of $100 \mu \mathrm{l}$ of an overnight $3 \mathrm{ml}$ culture in $3 \mathrm{ml}$ fresh BHI for a total of 50 days $(50 \times$ culture $)$. The $50 \times$ cultures were stored in $7 \%$ dimethyl sulfoxide at $-80{ }^{\circ} \mathrm{C}$ and their zeta potential distributions were measured as described above.

Anion-exchange chromatography. To enrich a bacterial culture for one subpopulation, a heterogeneous biliary stent isolate was passed through an anion-exchange column. The DEAE-Sephadex resin (Pharmacia) was preswollen in sterile $10 \mathrm{mM}$ potassium phosphate pH $7 \cdot 0$ and packed in a column $(5.5 \times 1.5 \mathrm{~cm}$ i.d.). The column was equilibrated with sterile $10 \mathrm{mM}$ potassium phosphate $\mathrm{pH} 7 \cdot 0$ and checked for sterility by addition of column material to fresh BHI; no growth appeared. A bacterial suspension in $30 \mathrm{ml} 10 \mathrm{mM}$ potassium phosphate $\mathrm{pH} 7 \cdot 0$ of $\mathrm{OD}_{600} 1.0$ was applied to the column, allowed to pass through by gravity and collected in $5 \mathrm{ml}$ fractions. A $100 \mu \mathrm{l}$ sample of each fraction was inoculated on a blood agar plate, after which the remainder of the fractions was added to $200 \mathrm{ml}$ fresh $\mathrm{BHI}$ and grown overnight at $37^{\circ} \mathrm{C}$. The zeta potential distributions were determined from the resulting cultures.

Quorum sensing. In order to analyse the effect of quorum sensing on heterogeneity, E. faecalis BS385 was grown in fresh and spent BHI. The supernatant of an overnight culture in BHI was passed through a $0.22 \mu \mathrm{m}$ pore-size syringe filter (Millipore) to yield a sterile conditioned medium that was subsequently added to fresh BHI to produce a final concentration of $50 \%$ fresh and $50 \%$ conditioned BHI (spent medium). The fresh BHI was twice concentrated to compensate for the loss of nutrients in the conditioned BHI. During the growth, $5 \mathrm{ml}$ aliquots were taken, cells washed twice with $10 \mathrm{mM}$ potassium phosphate $\mathrm{pH} 7 \cdot 0$ and the zeta potential distribution determined.

Effect of incubation in the presence of ox bile. Incubation in the presence of ox bile was performed with bacteria grown in BHI. The cells were incubated in $10 \mathrm{mM}$ potassium phosphate $\mathrm{pH} 7 \cdot 0$ with or without $50 \mathrm{mg}$ ox bile $\mathrm{ml}^{-1}$ for $24 \mathrm{~h}$ at $37^{\circ} \mathrm{C}$ and $4{ }^{\circ} \mathrm{C}$.
After washing the bacteria with $10 \mathrm{mM}$ potassium phosphate $\mathrm{pH} 7 \cdot 0$, the zeta potential distributions and water contact angles were measured, as described above.

Bacterial adhesion. A parallel-plate flow chamber $(175 \times 17 \times 0 \cdot 75$ $\mathrm{mm}$ ) was used to monitor bacterial adhesion as described previously (Waar et al., 2002c). Images were taken from the glass bottom plate. Bacteria from a $200 \mathrm{ml}$ overnight culture in BHI were harvested by centrifugation $\left(6500 \mathrm{~g}, 5 \mathrm{~min}, 10^{\circ} \mathrm{C}\right)$ and washed twice and resuspended in $10 \mathrm{mM}$ potassium phosphate $\mathrm{pH} 7 \cdot 0$, to a concentration of $3 \times 10^{8}$ cells $\mathrm{ml}^{-1}$. The bacterial suspension was allowed to flow through the system at a flow rate of $0.02 \mathrm{ml} \mathrm{s}^{-1}$ (shear rate $10 \mathrm{~s}^{-1}$ ) for $4 \mathrm{~h}$ with recirculation at room temperature. The adhesion to a negatively charged glass surface of a homogeneous strain, a heterogeneous strain and its enriched subpopulation obtained by anionexchange chromatography were compared.

The initial increase in number of adhering bacteria over time was expressed as the initial deposition rate $j_{0}$, while the number of bacteria adhering after $4 \mathrm{~h}, n_{4 \mathrm{~h}}$, was taken as an estimate of microbial adhesion in a more advanced state of the process. All adhesion experiments were performed in triplicate with separately cultured bacteria.

\section{RESULTS}

\section{Genetic analysis and the presence of esp and Agg}

Six E. faecalis strains were isolated from a total of 13 clogged biliary stents. PFGE patterns from these strains and RAPD analysis revealed close genetic relatedness between $E$. faecalis BS4126 and BS12297, whereas none of the other strains appeared closely related. All six isolates were evaluated for the presence of the enterococcal surface protein gene (esp) and only E. faecalis BS385 was esp deficient (Table 1). The expression of Aggs was determined with a clumping assay. Only E. faecalis BS12297 reacted to pheromones produced by $E$. faecalis $\mathrm{JH} 2-2$ and formed large aggregates indicating the expression of an Agg (Table 1). Interestingly, although

Table 1. Characteristics of stent-derived E. faecalis isolates

The presence of the esp gene was confirmed by PCR, and the presence of Agg was demonstrated in a clumping assay. Maximum growth rate was measured in BHI with or without ox bile. Water contact angles of the E. faecalis strains with and without ox bile during growth are presented as well.

\begin{tabular}{|c|c|c|c|c|c|c|}
\hline \multirow[t]{2}{*}{ E. faecalis } & \multirow[t]{2}{*}{$e s p^{*}$} & \multirow[t]{2}{*}{$\operatorname{Agg}^{\star}$} & \multicolumn{2}{|c|}{ Max. growth rate $\left(h^{-1}\right)$} & \multicolumn{2}{|c|}{ Contact angle (degrees) $\dagger$} \\
\hline & & & Without ox bile & With ox bile & Without ox bile & With ox bile \\
\hline BS4126 & + & - & 0.63 & $0 \cdot 54$ & 29 & $32 \ddagger$ \\
\hline BS12297 & + & + & $0 \cdot 61$ & $0 \cdot 47$ & 27 & $31 \neq$ \\
\hline BS385 & - & - & $0 \cdot 63$ & $0 \cdot 56$ & 32 & $22 \ddagger$ \\
\hline BS1037 & + & - & $0 \cdot 62$ & $0 \cdot 46$ & 30 & $27 \ddagger$ \\
\hline BS11297 & + & - & $0 \cdot 61$ & $0 \cdot 36$ & 28 & $23 \ddagger$ \\
\hline BS937 & + & - & $0 \cdot 54$ & $0 \cdot 38$ & 41 & $53 \neq$ \\
\hline
\end{tabular}

* + , Present; -, absent.

$\dagger$ The water contact angle was measured on three separate cultures, yielding a standard deviation of less than $15 \%$ of the mean.

$\ddagger P \leqslant 0 \cdot 05$ versus without ox bile, Student's $t$-test. 
PFGE and RAPD revealed that E. faecalis BS4126 and BS12297 showed close genetic relatedness, BS4126 did not aggregate, in contrast to BS12297, indicating that these two strains at least differ in the presence of an Agg in BS12297. Therefore, both E. faecalis BS4126 and BS12297 were included in this study.

\section{Cell surface hydrophobicity}

The cell surface hydrophobicity was assessed by water contact angle measurements. The water contact angles of all E. faecalis strains are summarized in Table 1. Five out of the six isolates can be classified as hydrophilic, while BS937 possessed an intermediate hydrophobicity, with a water contact angle of 41 degrees.

\section{Zeta potential distributions}

The $\mathrm{pH}$-dependent zeta potential distributions of the six E. faecalis cultures are presented in Fig. 1. Zeta potentials
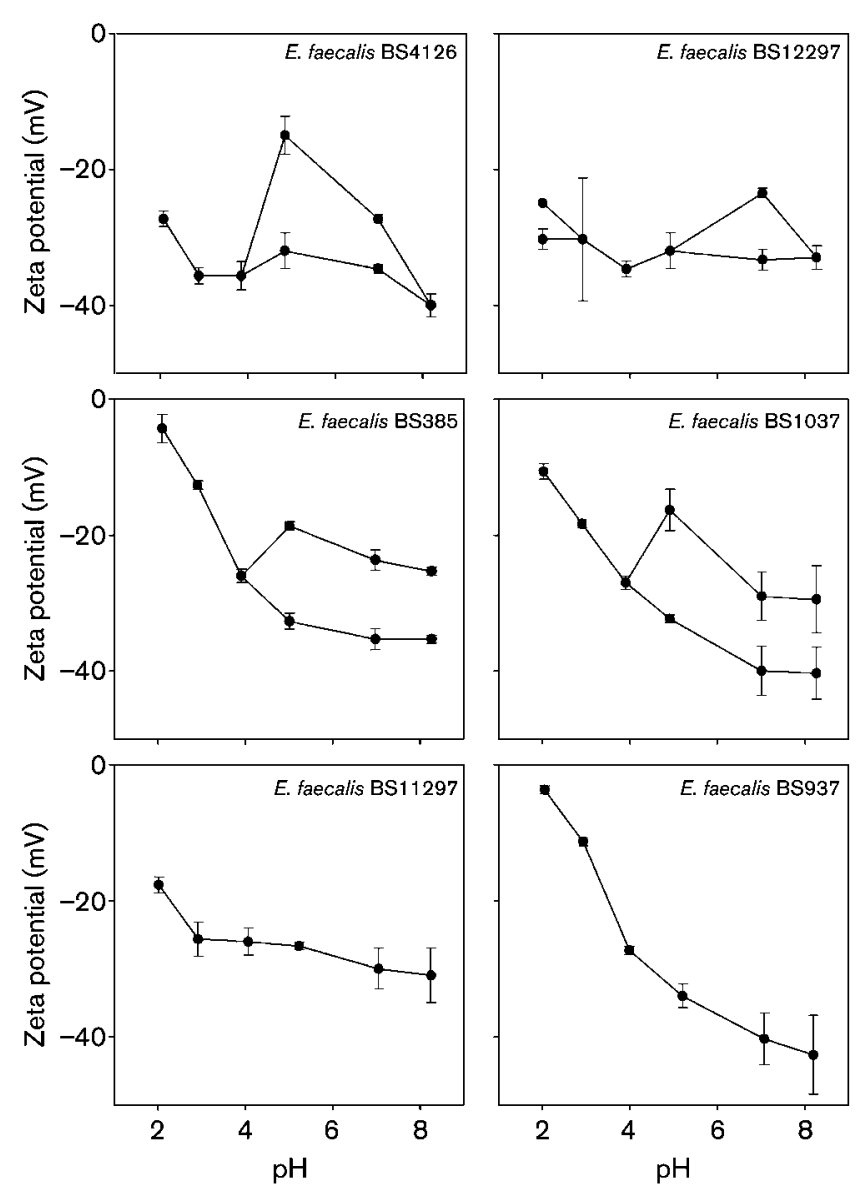

Fig. 1. Zeta potential distributions as a function of $\mathrm{pH}$ of $E$. faecalis BS4126, BS12297, BS385, BS1037, BS11297 and BS937, as determined in $10 \mathrm{mM}$ potassium phosphate $\mathrm{pH} 7 \cdot 0$. The bars denote standard deviations over three experiments, with each experiment comprising 100 bacteria. were negative for all strains over the entire $\mathrm{pH}$ range and four out of the six strains showed bimodal zeta potential distributions. Cultures of strains BS4126, BS12297, BS385 and BS1037 were heterogeneous with respect to their zeta potentials and at physiological $\mathrm{pH}$ value; these strains displayed two different zeta potentials.

The one strain that lacks the esp gene, BS385, is also heterogeneous in its zeta potential, indicating that the presence of esp is not involved in this phenomenon. This was confirmed by analysis of the zeta potential distributions of strains MMH594 (Esp $\left.{ }^{+}\right)$and MMH594b (Esp ${ }^{-}$), which differ only in the presence of the Esp protein. No significant difference in zeta potential distribution between these two strains was found (a table including the zeta potential distributions of MMH594 and MMH594b is provided as supplementary Table S1 with the online version of this paper).

Because one of the isolates was shown to possess an agg gene, the effect of Agg expression on the zeta potential distribution was analysed. When strain BS12297 was grown in the absence or presence of pheromone the zeta potential distribution was comparable. Identical results were obtained using E. faecalis OG1RF and OG1RF(pCF10), OG1X, OG1X(pAD1) and OG1X(pAM373), and FA2-2 and FA2-2(pAD1), indicating that Agg expression does not influence zeta potential distribution (see supplementary Table S1, which lists zeta potential distributions of enterococcal laboratory strains grown in the absence or presence of pheromone).

\section{Stability of heterogeneous subpopulations}

Serial passaging. To investigate whether culture heterogeneity was a stable trait, three heterogeneous strains and one homogeneous strain were serially passaged up to 50 times. Fig. 2 shows that the distributions of bacteria with a more or less negative zeta potential did not change significantly for the heterogeneous strains BS385, BS4126 and BS1037. The homogeneous strain BS937 did not change by serial passaging and continued to display a single population.

Anion-exchange chromatography. In an attempt to isolate one subpopulation, E. faecalis BS385 was passed through a DEAE-Sephadex column. Fractions from the column were grown in liquid medium and the zeta potentials of the cultures were determined. The percentage distribution of subpopulations in these cultures was inverted compared to the original culture (Fig. 3). Whereas the more negative subpopulation was in the minority prior to anion-exchange chromatography, this subpopulation presented a clear majority after anion-exchange chromatography. The fractions from the anion-exchange column were plated and individual colonies were subsequently cultured. However, no homogeneous cultures could be grown from up to 30 single colonies and the zeta potential distribution of all colonies invariably returned to the original distribution. 


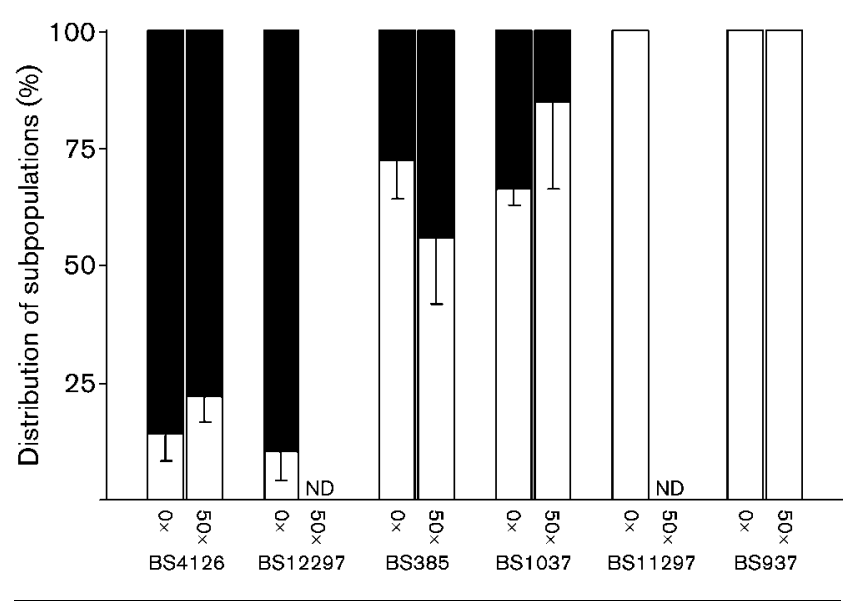

Fig. 2. Effect of 50 times serial passaging (indicated as $50 \times$ ) on the percentage distributions of subpopulations of $E$. faecalis BS4126, BS12297, BS385, BS1037, BS11297 and BS937 compared to the fresh clinical isolates. Distributions were determined by zeta potential measurements in $10 \mathrm{mM}$ potassium phosphate $\mathrm{pH} 7 \cdot 0$. In all cases the most negative fraction is indicated by black and the less negative fraction by white. The error bars denote standard deviations over three experiments, with each experiment comprising 100 bacteria. ND, Not determined.

Quorum sensing. Quorum sensing or regulated gene expression in response to culture density is mediated by small soluble molecules that are produced during growth and accumulate in the medium. The role of quorum sensing in culture heterogeneity was investigated by growing

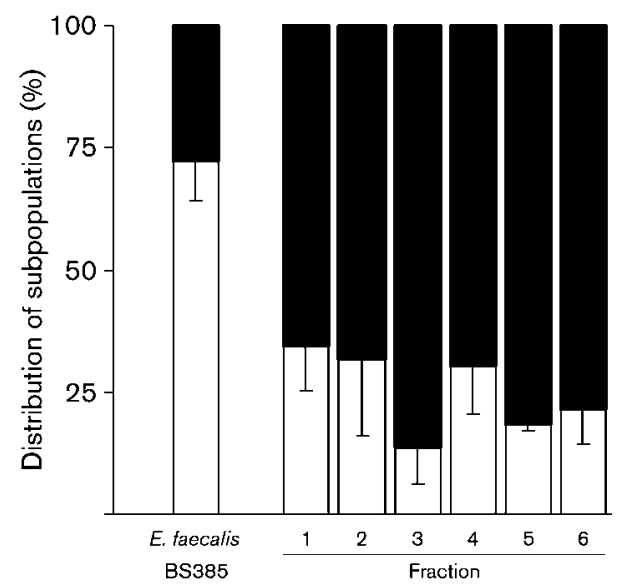

Fig. 3. Percentage distribution of subpopulations of $E$. faecalis BS385 culture grown from fractions passed through a DEAESephadex anion-exchange column. Distributions were determined by zeta potential measurements in $10 \mathrm{mM}$ potassium phosphate $\mathrm{pH} 7 \cdot 0$. In all cases the most negative fraction is indicated by black and the less negative fraction by white. The error bars denote standard deviations over three experiments, with each experiment comprising 100 bacteria.
E. faecalis BS385 in spent medium and comparing the zeta potential distribution with that of $E$. faecalis BS385 grown in fresh medium. The heterogeneity in zeta potential was determined every 30 min during growth and was found to be the same, within the margin of error, during the whole growth phase, i.e. the strain was always heterogeneous, independent of growth phase (see supplementary Table S2 for zeta potential distributions of BS385 during growth). The presence of spent medium did not influence the zeta potential distribution. In addition, growing $E$. faecalis BS385 in either another rich medium (tryptone soya broth) or minimal medium (M9) did not affect the subpopulation distribution.

Growth and incubation in the presence of ox bile. The maximum growth rates of the E. faecalis strains grown in the presence of ox bile were reduced by a factor of 1.7 (BS11297) to $1 \cdot 1$ (BS385) compared to the maximum growth rates of the strains grown without ox bile (Table 1). The total numbers of bacteria in the stationary phase were lower when bacteria were grown in the presence of ox bile. Three strains became slightly more hydrophobic when grown in the presence of ox bile, while the other three strains became more hydrophilic (see Table 1). After growth in the presence of ox bile, however, culture heterogeneity disappeared and all strains displayed one population (see Table 2).

In order to gain more insight into the effect of ox bile on culture heterogeneity, E. faecalis BS385 grown in the absence of ox bile was incubated in $10 \mathrm{mM}$ potassium phosphate $\mathrm{pH} 7$ in the absence or presence of ox bile. Irrespective of the incubation temperature $\left(4^{\circ} \mathrm{C}\right.$ or $\left.37^{\circ} \mathrm{C}\right)$, the culture heterogeneity persisted after incubation in ox bile and only disappeared when the bacterium was actually grown in the presence of bile. The water contact angle was not affected by incubation in ox bile.

\section{Bacterial adhesion}

Table 3 compares the adhesion to a glass surface of the heterogeneous strain E. faecalis BS385, its subpopulation

Table 2. Zeta potential of $E$. faecalis strains grown in $\mathrm{BHI}$ in the presence of ox bile, as determined in $10 \mathrm{mM}$ potassium phosphate $\mathrm{pH} 7 \cdot 0$

The results are mean \pm SD of three separate experiments performed in triplicate, with each experiment comprising 100 bacteria.

\begin{tabular}{|lc|}
\hline E. faecalis & Zeta potential $(\mathbf{m V})$ \\
\hline BS4126 & $-32 \pm 3$ \\
BS12297 & $-32 \pm 2$ \\
BS385 & $-35 \pm 1$ \\
BS1037 & $-29 \pm 3$ \\
BS11297 & $-28 \pm 3$ \\
BS937 & $-34 \pm 4$ \\
\hline
\end{tabular}


Table 3. Adhesion of various E. faecalis populations

Initial deposition rates and number of bacteria adhering to a negatively charged glass surface after $4 \mathrm{~h}$ are shown for heterogeneous E. faecalis BS385 culture, its isolated, more negatively charged subpopulation and homogeneous culture BS11297.

\begin{tabular}{|lcc|}
\hline & $\boldsymbol{j}_{\mathbf{0}}^{*}$ & $\boldsymbol{n}_{\mathbf{4 h}}{ }^{\dagger}$ \\
\hline Heterogeneous strain E. faecalis BS385 & $691 \pm 135$ & $8 \cdot 0 \pm 0 \cdot 9$ \\
Enriched subpopulation of E. faecalis BS385 & $240 \pm 28 \ddagger$ & $3 \cdot 5 \pm 0 \cdot 3 \ddagger$ \\
Homogeneous strain E. faecalis BS11297 & $825 \pm 172$ & $0 \cdot 5 \pm 0 \cdot 1 \ddagger$ \\
\hline
\end{tabular}

${ }^{*} j_{0}$, initial deposition rate $\left(\mathrm{cm}^{-2} \mathrm{~s}^{-1}\right)$.

$\dagger n_{4 \mathrm{~h}}$, number of bacteria adhering after $4 \mathrm{~h}\left(10^{6} \mathrm{~cm}^{-2}\right)$.

$\ddagger P \leqslant 0 \cdot 05$ versus $E$. faecalis BS385, non-parametric Mann-Whitney $U$-test.

enriched in the more negatively charged bacteria and homogeneous strain E. faecalis BS11297. Although the initial deposition rate was highest for the homogeneous strain, 16 times less bacteria adhered after $4 \mathrm{~h}$ from the homogeneous strain than from the heterogeneous strain. The initial deposition rate and the number of adhering bacteria after $4 \mathrm{~h}$ were both significantly higher for the heterogeneous strain than for the more negatively charged subpopulation.

\section{Incidence of heterogeneity in $E$. faecalis isolates}

Heterogeneity in zeta potential was not limited to E. faecalis isolated from clogged biliary stents, but was also present in other clinical isolates. Among 29 E. faecalis strains isolated from faeces of healthy volunteers, blood from patients suffering E. faecalis bacteraemia, and liver patients, 13 showed culture heterogeneity in zeta potential (see Table 4). Frequently used $E$. faecalis laboratory strains also showed heterogeneity in zeta potential (see Table 4). Thus, culture heterogeneity appears to be a common trait among E. faecalis strains, irrespective of the presence of esp or Agg (see also Table 4).

\section{DISCUSSION}

Upon analysis of factors involved in adhesion among $E$. faecalis strains isolated from clogged biliary stents, culture heterogeneity in zeta potential was observed. Four out of six strains were heterogeneous, and about half of all other clinical isolates and laboratory strains analysed showed heterogeneity in their zeta potential. There was no correlation between the observed heterogeneity and cell surface hydrophobicity or the presence of esp or Agg. Culture heterogeneity was not dependent on the medium or carbon source used for culturing, nor on growth phase or quorum sensing. The heterogeneous strains became homogeneous upon culturing in the presence of ox bile. Analysis of the hydrophobicity showed that this resulted in increased hydrophobicity for three strains while the other three strains became more hydrophilic. This is in accordance with previous findings that four E. faecalis strains became more hydrophobic after growth in the presence of ox bile (Waar et al., 2002b). Simple exposure by incubation in ox bile without growth did not affect the hydrophobicity or the zeta potential distribution. This shows that the changes in surface properties after growth in the presence of ox bile are not caused by adsorption of bile salts to the cell surface, but by a change in physiology of the bacteria in response to bile.

Table 4. Occurrence of heterogeneity in zeta potential, and presence of esp and Agg in E. faecalis strains of different origin

Strain listing and measured values are provided in supplementary Tables S1 and S4.

\begin{tabular}{|lccc|}
\hline Origin (no. of isolates) & $\begin{array}{c}\text { Heterogeneous/homogeneous } \\
\text { strains }\end{array}$ & $\begin{array}{c}\text { esp positive } \\
\text { (heterogeneous/homogeneous) }\end{array}$ & $\begin{array}{c}\text { Agg positive } \\
\text { (heterogeneous/homogeneous) }\end{array}$ \\
\hline Faeces of healthy volunteers (9) & $3 / 6$ & $1 / 4^{\star}$ & $2 / 4^{\star}$ \\
Liver transplant patients (10) & $4 / 6$ & $1 / 5^{\star}$ & $3 / 3^{\star}$ \\
Blood cultures (10) & $6 / 4$ & $2 / 4^{\star}$ & $4 / 2^{\star}$ \\
Biliary stents (6) & $4 / 2$ & $3 / 2$ & $1 / 0$ \\
Lab strains (11) & $7 / 4$ & $0 / 1$ & $3 / 4$ \\
Total (46) & $24 / 22$ & $7 / 16$ & $13 / 13$ \\
\hline
\end{tabular}

*Taken from Waar et al. (2002a). 
Five of the six E. faecalis strains isolated from clogged biliary stents were esp-positive, which is in accordance with a previous study where it was shown that esp was significantly enriched in infection-derived enterococcal strains (Shankar et al., 1999). There was no correlation between the presence of esp and heterogeneity in zeta potential, which is confirmed by the fact that the zeta potential distribution of MMH594 (Esp ${ }^{+}$) and MMH594b (Esp $\left.{ }^{-}\right)$is not significantly different. Donelli et al. (2004) found that Agg might be one of the most important causative factors in the clogging of biliary stents. Six out of twelve E. faecalis isolated from clogged biliary stents were Agg-positive (Donelli et al., 2004). Expression of Agg enhanced the adhesion of E. faecalis strains to bile drain materials through positive cooperativity (Waar et al., 2002c) and it has been shown that Agg is expressed in vivo (Hirt et al., 2002). However, in the present study, only one of the six isolates reacted to pheromones indicating the presence of an Agg, and no correlation between the presence or absence of Agg and heterogeneity in zeta potential was present.

Culture heterogeneity in zeta potential is seen for bacteria isolated from different environments, ranging from the human oral cavity (Cowan et al., 1992) to freshwater reservoirs (Streger et al., 2002). The results presented here indicate that heterogeneity in zeta potential is a common trait of E. faecalis and independent of the origin of the isolate (see Table 4). Two discrete electrophoretic mobilities in a pure culture of $E$. faecalis have been previously found (Ebersole \& McCormick, 1993) and were suggested to be due to differences in chain assemblage, i.e. single cells and dimers versus longer chains. However, in the present study the cell suspensions were not routinely sonicated to break chains into single cells or dimers, because experiments had indicated that sonication had no effect on the zeta potential distributions (see supplementary Fig. S1 for the effect of sonication on the chain length and supplementary Table S3 for the effect of sonication on the zeta potential distributions). Therefore we conclude that culture heterogeneity is due to intrapopulation variance in cell surface charge and not due to possible variations in chain length.

It was possible to enrich one subpopulation using affinity chromatography. The zeta potential distributions of single colonies from the enriched subpopulation invariably returned to the original distribution after growth in liquid medium, indicating that the difference in zeta potential is not caused by a mutation in part of the culture. It is our hypothesis that differential gene expression during culture is the basis for the observed heterogeneity, in analogy to the development of competence in Bacillus subtilis (reviewed by Dubnau, 1991; Hamoen et al., 2003). It is interesting that only one-third of the faeces-derived isolates showed heterogeneity, while two-thirds of either blood- or stentderived isolates that had been involved in infection showed culture heterogeneity in their zeta potentials. Further studies are necessary to establish the putative role of culture heterogeneity in the pathogenicity of E. faecalis.
An important enterococcal virulence factor is adhesion. In this study we found that heterogeneous strains adhered significantly better to glass surfaces than homogeneous strains. Note that glass was used, as it has a negative surface charge that is comparable to polyethylene (a biliary stent material) $(-75 \mathrm{mV}$ and $-70 \mathrm{mV}$, respectively, in $1 \mathrm{mM}$

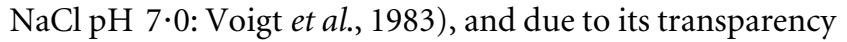
it is readily applicable in the parallel-plate flow chamber. In addition, heterogeneous strains present two different surface charges to their environment, allowing them to adhere to surfaces with different surface properties, thus increasing their chances of successful colonization of different biomedical surfaces.

In conclusion, two-thirds of the biliary stent isolates showed culture heterogeneity in cell surface charge, which is a common phenomenon among $E$. faecalis strains. Culture heterogeneity offers the organism an advantage in adhesion to a negatively charged surface. Interestingly, two-thirds of the pathogenic isolates of $E$. faecalis examined showed culture heterogeneity in zeta potential, while this was true for only one-third of the faecal isolates. Future research will focus on identification of processes regulating the observed heterogeneity in zeta potential and further investigate the role of this phenomenon in virulence and pathogenicity.

\section{ACKNOWLEDGEMENTS}

We thank Professor Dr G. M. Dunny for providing E. faecalis OG1RF and OG1RF(pCF10).

\section{REFERENCES}

Barbier, N., Saulnier, P., Chachaty, E., Dumontier, S. \& Andremont, A. (1996). Random amplified polymorphic DNA typing versus pulsedfield gel electrophoresis for epidemiological typing of vancomycinresistant enterococci. J Clin Microbiol 34, 1096-1099.

Chow, J. W., Thal, L. A., Perri, M. B., Vazquez, J. A., Donabedian, S. M., Clewell, D. B. \& Zervos, M. J. (1993). Plasmid-associated hemolysin and aggregation substance production contribute to virulence in experimental enterococcal endocarditis. Antimicrob Agents Chemother 37, 2474-2477.

Clewell, D. B. (1993). Bacterial sex pheromone-induced plasmid transfer. Cell 73, 9-12.

Clewell, D. B., Tomich, P. K., Gawron-Burke, M. C., Franke, A. E., Yagi, Y. \& An, F. Y. (1982). Mapping of Streptococcus faecalis plasmids pAD1 and pAD2 and studies relating to transposition of Tn917. J Bacteriol 152, 1220-1230.

Clewell, D. B., An, F. Y., White, B. A. \& Gawron-Burke, M. C. (1985). Streptococcus faecalis sex-pheromone (cAM373) also produced by Staphylococcus aureus and identification of a conjugative transposon (Tn918). J Bacteriol 162, 1212-1220.

Cowan, M. M., Van der Mei, H. C., Stokroos, I. \& Busscher, H. J. (1992). Heterogeneity of surfaces of subgingival bacteria as detected by zeta potential measurements. J Dent Res 71, 1803-1806.

Di Rosa, R., Basoli, A., Donelli, G., Penni, A., Salvatori, F. M., Fiocca, F. \& Baldassarri, L. (1999). A microbiological and morphological study of blocked biliary stents. Microb Ecol Health Dis 11, 84-88. 
Donelli, G., Paoletti, C., Baldassarri, L., Guablianone, E., Di Rosa, R., Magi, G., Spinaci, C. \& Facinelli, B. (2004). Sex pheromone response, clumping, and slime production in enterococcal strains isolated from occluded biliary stents. J Clin Microbiol 42, 3419-3427.

Dowidar, N., Kolmos, H. J., Lyon, H. \& Matzen, P. (1991). Clogging of biliary endoprostheses. A morphologic and bacteriologic study. Scand J Gastroenterol 26, 1137-1144.

Dubnau, D. (1991). Genetic competence in Bacillus subtilis. Microbiol Rev 55, 395-424.

Dunny, G. M., Brown, B. L. \& Clewell, D. B. (1978). Induced cell aggregation and mating in Streptococcus faecalis: evidence for a bacterial sex pheromone. Proc Natl Acad Sci U S A 75, 3479-3483.

Dunny, G. M., Funk, C. \& Adsit, J. (1981). Direct stimulation of the transfer of antibiotic-resistance by sex-pheromones in Streptococcus faecalis. Plasmid 6, 270-278.

Ebersole, R. C. \& McCormick, R. M. (1993). Separation and isolation of viable bacteria by capillary zone electrophoresis. Biotechnology 11, $1278-1282$.

Geertsema-Doornbusch, G. I., Noordmans, J., Bruce, A. W., Reid, G., Khoury, A. E., Van der Mei, H. C. \& Busscher, H. J. (1994). Quantification of microbial cell surface heterogeneity by microelectrophoresis and electron microscopy - application to lactobacilli after serial passaging. J Microbiol Methods 19, 269-277.

Glynn, J. R., Belongia, B. M., Arnold, R. G., Ogden, K. L. \& Baygents, J. C. (1998). Capillary electrophoresis measurements of electrophoretic mobility for colloidal particles of biological interest. Appl Environ Microbiol 64, 2572-2577.

Hamoen, L. W., Venema, G. \& Kuipers, O. P. (2003). Controlling competence in Bacillus subtilis: shared use of regulators. Microbiology 149, 9-17.

Hirt, H., Schlievert, P. M. \& Dunny, G. M. (2002). In vivo induction of virulence and antibiotic resistance transfer in Enterococcus faecalis mediated by the sex pheromone-sensing system of pCF10. Infect Immun 70, 716-723.

Ike, Y., Craig, R. A., White, B. A., Yagi, Y. \& Clewell, D. B. (1983). Modification of Streptococcus faecalis sex-pheromones after acquisition of plasmid DNA. Proc Natl Acad Sci U S A 80, 5369-5373.

Jacob, A. E. \& Hobbs, S. J. (1974). Conjugal transfer of plasmidborne multiple antibiotic resistance in Streptococcus faecalis var. zymogenes. J Bacteriol 117, 360-372.

Kayaoglu, G. \& Østavik, D. (2004). Virulence factors of Enterococcus faecalis: relationship to endodontic disease. Crit Rev Oral Biol Med 15, 308-320.

Kell, D. B., Ryder, H. M., Kaprelyants, A. S. \& Westerhoff, H. V. (1991). Quantifying heterogeneity: flow cytometry of bacterial cultures. Antonie van Leeuwenhoek 60, 145-158.

Low, D. E., Keller, N., Barth, A. \& Jones, R. N. (2001). Clinical prevalence, antimicrobial susceptibility, and geographic resistance patterns of enterococci: results from the SENTRY Antimicrobial Surveillance Program, 1997-1999. Clin Infect Dis 32, S133-S145.

Murray, B. E., Singh, K. V., Heath, J. D., Sharma, B. R. \& Weinstock, G. M. (1990). Comparison of genomic DNAs of different enterococcal isolates using restriction endonucleases with infrequent recognition sites. J Clin Microbiol 28, 2059-2063.

Muscholl, A., Galli, D., Wanner, G. \& Wirth, R. (1993). Sex pheromone plasmid pAD1-encoded aggregation substance of Enterococcus faecalis is positively regulated in trans by traE1. Eur J Biochem 214, 333-338.

Noordmans, J., Kempen, J. \& Busscher, H. J. (1993). Automated image analysis to determine zeta potential distributions in particulate microelectrophoresis. J Colloid Interface Sci 156, 394-399.

Shankar, V., Baghdayan, A. S., Huycke, M. M., Lindahl, G. \& Gilmore, M. S. (1999). Infection-derived Enterococcus faecalis strains are enriched in esp, a gene encoding a novel surface protein. Infect Immun 67, 193-200.

Shankar, N., Baghdayan, A. S. \& Gilmore, M. S. (2001a). Modulation of virulence within a pathogenicity island in vancomycinresistant Enterococcus faecalis. Nature 417, 746-750.

Shankar, N., Lockatell, C. V., Baghdayan, A. S., Drachenberg, C., Gilmore, M. S. \& Johnson, D. E. (2001b). Role of Enterococcus faecalis surface protein Esp in the pathogenesis of ascending urinary tract infection. Infect Immun 69, 4366-4372.

Streger, S. H., Vainberg, S., Dong, H. L. \& Hatzinger, P. B. (2002). Enhancing transport of Hydrogenophaga flava ENV735 for bioaugmentation of aquifers contaminated with methyl tert-butyl ether. Appl Environ Microbiol 68, 5571-5579.

Tendolkar, P. M., Baghdayan, A. S., Gilmore, M. S. \& Shankar, N. (2004). Enterococcal surface protein, Esp, enhances biofilm formation by Enterococcus faecalis. Infect Immun 72, 6032-6039.

Toledo-Arana, A., Valle, J., Solano, C. \& 7 other authors (2001). The enterococcal surface protein, Esp, is involved in Enterococcus faecalis biofilm formation. Appl Environ Microbiol 67, 4538-4545.

Van der Mei, H. C. \& Busscher, H. J. (2001). Electrophoretic mobility distibutions of single-strain microbial populations. Appl Environ Microbiol 67, 491-494.

Van Loosdrecht, M. C. M., Lyklema, J., Norde, W., Schraa, G. \& Zehnder, A. J. B. (1987). Electrophoretic mobility and hydrophobicity as a measure to predict the initial steps of bacterial adhesion. Appl Environ Microbiol 53, 1898-1901.

Van Oss, C. J. (2003). Long-range and short-range mechanisms of hydrophobic attraction and hydrophilic repulsion in specific and aspecific interactions. J Mol Recognit 16, 177-190.

Voigt, A., Wolf, H., Lauekner, S., Neumann, G., Becker, R. \& Richter, L. (1983). Electrokinetic properties of polymer and glass surfaces in aqueous solutions: experimental evidence for swollen surface layers. Biomaterials 4, 299-304.

Waar, K., Muscholl-Silberhorn, A. B., Willems, R. J. L., Slooff, M. J. H., Harmsen, H. J. M. \& Degener, J. E. (2002a). Genogrouping and incidence of virulence factors of Enterococcus faecalis in liver transplant patients differ from blood culture and fecal isolates. J Infect Dis 185, 1121-1127.

Waar, K., Van der Mei, H. C., Harmsen, H. J. M., Degener, J. E. \& Busscher, H. J. (2002b). Adhesion to bile drain materials and physicochemical surface properties of Enterococcus faecalis strains grown in the presence of bile. Appl Environ Microbiol 68, 3855-3858.

Waar, K., Van der Mei, H. C., Harmsen, H. J. M., Degener, J. E. \& Busscher, H. J. (2002c). Enterococcus faecalis surface proteins determine its adhesion mechanism to bile drain materials. Microbiology 148, 1863-1870.

Wilson, W. W., Wade, M. M., Holman, S. C. \& Champlin, F. R. (2001). Status of methods for assessing bacterial cell surface charge properties based on zeta potential measurements. J Microbiol Methods 43, 153-164. 